\title{
Control of conversion and separation of lignocellulose components by phase-separation conditions
}

\author{
Keigo Mikame and Masamitsu Funaoka \\ SORST JST, Graduate School of Bioresources, Mie University, 1577, Kurima-machiya, Tsu, Mie 514-8507, Japan \\ Fax:+81-59-231-9517, E-mail: mikame@bio.mie-u.ac.jp
}

\begin{abstract}
Through the phase-separation reaction system composed of phenol derivatives and concentrated acid, lignin and carbohydrates, which form an interpenetrating polymer network within the cell wall, were separated almost quantitatively into different phases within $20 \mathrm{~min}$. The yield of carbohydrate of acid layer after the 60 minutes phase-separation treatment was $71 \%$ of raw material $(98 \%$ of carbohydrate of raw material). A total yield of original lignin without grafting cresol in lignocresol, acid phase sugar and cresol phase sugar was $96 \%$ of raw material at the 60 minutes phase-separation treatment. It was possible to control conversion pattern of lignocellulosics component with the quantity of the phenol derivatives and the hydrophilicity of the phenol derivatives.

Separated carbohydrates were consisted of various sizes of oligosaccharide and high molecular weight water-soluble glucose polymers. In the phase-separation treatments with different hydrophobic phenol derivatives, the separation efficiency of lignocellulocics got higher with increasing hydrophilicity of the phenol became high because of the affinity of the phenol phase and acid phase.

Key words: lignin, cellulose, hemicellulose, lignophenol, phase-separation system
\end{abstract}

\section{INTRODUCTION}

In recent years, lignocellulosic materials have shown interesting features to be used as raw materials for industrial material production. However, the lignocellulosics must be separated into individual components before use as chemicals. Separating lignocellulosic components is extremely difficult, since the lignocellulosic have the interpenetrating polymer network structure composed of lignin, hemicellulose and cellulose. So far the lignocellulosics have been treated with strong alkaline for paper making or hydrolyzed with strong acid for pretreatment of ethanol fermentation. Lignins are subjected to very complicated modifications and deactivated during these treatments, ${ }^{1-3}$ thus denatured lignin have been treated as if a waste.

Recently a new process for separating lignocellulosics into lignin and carbohydrate moieties with the conversion to highly reactive forms at room temperature was developed. ${ }^{4}$ This process includes a phase-separative reaction system composed of phenol derivatives and concentrated acid which are immiscible with each other. In the process the concentrated acid is not only a solvent for carbohydrate, but also works as a catalyst for the fragmentation and phenolation of lignin, whereas phenol derivatives act as phenolation agents, a barrier to minimize the attack of acid on the lignin and a solvent for the lignin fractions. The separated lignin derivatives (lignophenol) have several unique functions, which conventional lignins do not have, in spite of retention of the original interunit linkages: these include highly phenolic property, no conjugated system, light color comparable with native lignin, solid-liquid transformation and high immobilization capability for proteins. ${ }^{5,6}$ These original functions are due to the selective hybridization of monomeric phenol derivatives at C-1 positions, leading to linear type structures composed mainly of 1,1-bis(aryl)propane-type units.

In this study, conversion and separation pattern of plant cell wall components through the phase-separation system was investigated. The composition and molecular weight distribution of separated carbohydrates and material balance of lignocellulosic components were examined with various condition of phase-separation treatment. The conversion and separation pattern of lignin component was reported in previous papers.

\section{EXPERIMENTAL}

2.1 Phase-separation treatment 1 step process $p$-Cresol $(10 \mathrm{ml} / \mathrm{g}$ wood) was added to spruce wood meals with stirring at room temperature. After $10 \mathrm{~min}, 72 \%$ sulfuric acid $(15 \mathrm{ml} / \mathrm{g}$ wood $)$ was added to the mixture and the vigorous stirring was continued at $30^{\circ} \mathrm{C}$ for the prescribed time.

The reaction mixture was rapidly separated into the organic and aqueous phases by centrifugation. The organic phase was taken up and added dropwise to an excess amount of ethyl ether with vigorous stirring. The precipitates were dissolved in acetone and insoluble materials were removed 
by centrifugation. The acetone solution was then concentrated under reduced pressure and added dropwise to an excess amount of ethyl ether with stirring. The precipitated lignin derivative (lignocresol) was collected by centrifugation.

2.2 Phase-separation treatment 2 step process

For solvation of lignin with phenol derivatives, 3 $\mathrm{mol} / \mathrm{C} 9$ of $p$-cresol dissolved in acetone was added to wood meal and acetone was evaporated with stirring. Sulfuric acid $(72 \% 15 \mathrm{ml} / \mathrm{g}$ wood) was added to the mixture and the vigorous stirring was continued at $30^{\circ} \mathrm{C}$ for the prescribed time. Ninety seconds before a reaction end time, $5 \mathrm{ml}$ of $p$-cresol was added to reaction mixture. After stirring for 90 seconds, the reaction mixture was rapidly separated into the organic and aqueous phases by centrifugation. Extraction and purification of lignocresol from organic phase was preformed as well as 1 step process.

2.3 Molecular weight distribution of lignocresol

Size exclusion chromatogram of lignocresol was determined on a JASCO PU-1580 equipped with JASCO MD-1515 multi wavelength detector. Shodex GPC KF-801,802,803,804 (8 mm ID x 300 $\mathrm{mm}$ ) was connected in series and THF used as an eluent [flow rate; $1.0 \mathrm{ml} / \mathrm{min}$ ].

2.4 Carbohydrate composition

The aqueous phase after phase-separation treatment was diluted to $3 \%$ sulfuric acid concentration with distilled water, and was boiled for 4 hours. After cooling, ribose as an internal standard was added. The solution was neutralized and desalted by barium hydroxide, and was analyzed by the SHIMADZU HPLC Reducing Sugar Analysis System.

(HPLC condition)

Column: Shim-pack ISA-07/S2504 (4mm ID. $\times 25$ $\mathrm{cm} \mathrm{L.} \mathrm{).} \mathrm{Eluent:} \mathrm{A;} 0.1 \mathrm{M}$ potassium borate buffer $(\mathrm{pH} 8.0), \quad \mathrm{B} ; 0.4 \mathrm{M}$ potassium borate buffer (pH9.0), Flow rate: $0.6 \mathrm{ml} / \mathrm{min}$. Gradient: A $100 \%$ to B $100 \%(2 \% / \mathrm{min})$. Temp: $65^{\circ} \mathrm{C}$. Detect; reaction reagent; $1 \% L$-arginine, $3 \%$ Boric acid, $0.5 \mathrm{ml} / \mathrm{min}$. Reaction temp.; $150^{\circ} \mathrm{C}$, detection wave; Ex-320 nm, Em-430 nm

The composition of carbohydrate in the organic phase was analyzed as follows. The organic phase was added to diethyl ether with stirring. The precipitates were dissolved in acetone and the insoluble materials were collected by centrifugation. The insoluble materials $(10 \mathrm{mg})$ were treated with $1 \mathrm{ml}$ of $72 \%$ sulfuric acid at $30^{\circ} \mathrm{C}$ for 1 hour. The reaction mixture was diluted to $3 \%$ sulfuric acid concentration with distilled water and heated in an autoclave at $120^{\circ} \mathrm{C}$ for 1 hour. After cooling, ribose as an internal standard was added. After neutralization and desalting, the supernatant was analyzed by the same method as the aqueous phase.

2.5 Molecular weight distribution of carbohydrates

The aqueous phase after sulfuric acid hydrolysis was neutralized and desalted by barium hydroxide. The solution was analyzed by gel permeation chromatography (GPC).

(GPC condition)

Column: Asahipak GS-620HQ, GS-520HQ, GS-320HQ, GS-220HQ (7.6 mm ID. $\times 30 \mathrm{~cm} \mathrm{~L}$ )

Eluent: $\mathrm{H}_{2} \mathrm{O}$. Flow rate: $0.6 \mathrm{~m} 1 / \mathrm{min}$. Temp: $50^{\circ} \mathrm{C}$.

Detect: RI. Standard: Pullulan, maltose, maltotriose, maltopentaose, maltoheptaose 2.6 Contents of combined cresol of lignocresols

${ }^{1} \mathrm{H}-\mathrm{NMR}$ analysis of fractionated lignocresols were performed on a JEOL JNM-A500 FT-NMR System. The amount of cresol induced was calculated based on the signal intensity of cresolic methyl protons $(1.6-2.4 \mathrm{ppm})$ against aromatic protons $(7.8-8.4 \mathrm{ppm})$ of $p$-nitrobenzaldehyde (internal standard) on ${ }^{1} \mathrm{H}-\mathrm{NMR}$ spectra.

\section{RESULTS AND DISCUSSION}

3.1 Carbohydrate composition

Figure 1 shows the carbohydrate composition of the aqueous phase after the 1 step phase separation treatment. With phase separation treatment for 5 min, the content of hemicellulose sugar (mannose, arabinose, galactose, xylose) was about $20 \%$ of raw material, corresponding to $31 \%$ of total neutral sugar in the sulfuric acid phase. However, the content of hemicellulose sugar in the aqueous phase was about $19 \%$ of total neutral sugar after $60 \mathrm{~min}$. This reason is that noncrystalline hemicellulose is preferentially hydrolyzed at the early step of phase-separation treatment, followed by hydrolysis of cellulose. Total neutral sugar from the sulfuric acid phase after the phase separation treatment was $62 \%$ of raw material ( $86 \%$ of total carbohydrate) in 5 min treatment time, and the neutral sugar in the sulfuric acid phase increased with the treatment, the content was $71 \%$ of raw material $(98 \%$ of total

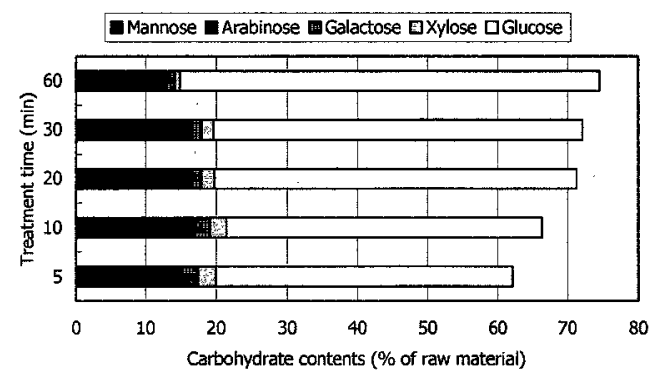

Fig. 1. Carbohydrate compositions of the sulfuric acid phase after the lstep phase-separation treatment

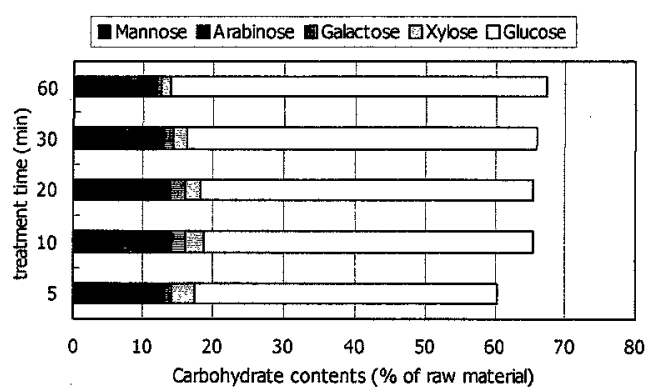

Fig. 2. Carbohydrate compositions of the sulfuric acid phase after the 1 step phase-separation treatment 
carbohydrate) in $20 \mathrm{~min}$ treatment and the content was $75 \%$ (104\% of total carbohydrate) in $60 \mathrm{~min}$ treatment.

Figure 2 shows the carbohydrate composition of the aqueous phase after the 2 step phase separation treatment. Total neutral sugar from the sulfuric acid phase after the 2step phase separation treatment was less than the 1 step phase separation treatment; the yields were $65 \%$ of raw material (90\% of total carbohydrate) in $20 \mathrm{~min}$ treatment and the content was $68 \% \quad(95 \%$ of total carbohydrate) in $60 \mathrm{~min}$ treatment.

Because the $p$-cresol works not only as conversion agent for lignin, but also as solvents for extracting the resulting lignocresol, the lignocresol is efficiently separated from lignocellulosics. Thus the belt effect was inhibited and hydrolysis of cellulose was accelerated.

On the other hand, the content of carbohydrate of the cresolic phase was getting decreased with the treatment time; $1.8 \%$ of raw material at $5 \mathrm{~min}$ and $0.7 \%$ of raw material at $60 \mathrm{~min}$ of the 1 step phase separation treatment. The content of hemicellulose sugar was $40 \%$ of total neutral sugar in the cresolic phase at $5 \mathrm{~min}$ of the phase separation treatment. Because a part of lignin is linked to hemicellulose, the hemicellulose was separated into the cresolic phase with lignocresol at an early stage of phase separation treatment. However, the content of hemicellulose sugar was decreased to $18 \%$ after $60 \mathrm{~min}$ treatment, because the linkages between lignin and hemicellulose were cleaved through the contact with acid at the interface between two phases and hemicellulose sugar was transported to the aqueous phase. The content of carbohydrate of the cresolic phase after the 2 step phase separation treatment was less than the 1step phase separation treatment; $0.15 \%$ of raw material at $60 \mathrm{~min}$ treatment. The ratio of hemicellulose sugar in cresolic phase carbohydrates after the 2 step phase separation treatment was higher than the 1step phase separation treatment. Because the transferable carbohydrate to cresolic phase is difficult for low content of phenol derivatives, and the carbohydrate which can shift on this condition was restricted almost exclusively to affinity carbohydrate to lignin for example lignin-carbohydrate complex (LCC).

3.2 Molecular weight distribution of carbohydrates

The carbohydrates in the sulfuric acid phase were consisted of high molecular weight fractions (more than $\mathrm{Mw}=100,000$ ) and low molecular weight fraction (less than $\mathrm{Mw}=2,000$ ) (Fig. 3, 4).

The high molecular weight fraction was $65 \%$ of the acid phase carbohydrate after the $10 \mathrm{~min} 1 \mathrm{step}$ phase-separation treatment. The high molecular weight fraction was decreased to $60 \%$ of the acid phase carbohydrate after the treatment time 60 min. When treatment time was extended till 240 $\mathrm{min}$, the ratio of the fraction was decreased to $24 \%$ after the $240 \mathrm{~min}$, although it doesn't show
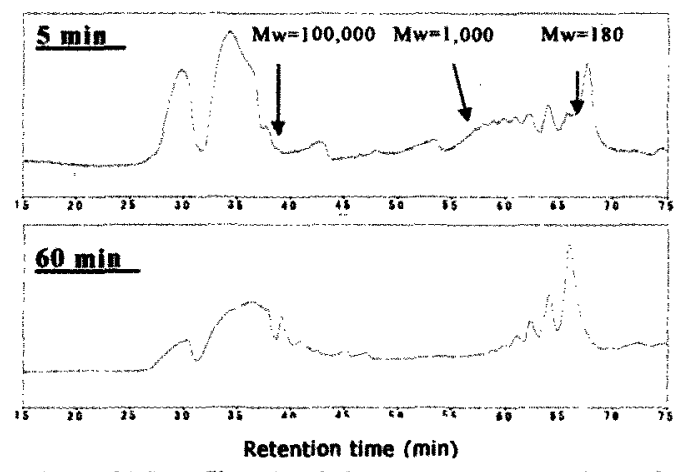

Fig. 3. GPC profiles of carbohydrates in the acid phase after 1step phase-separation treatment
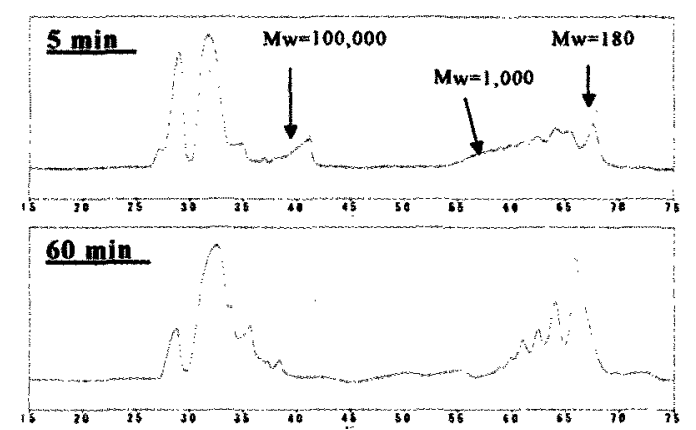

Retention time ( $\mathrm{min})$

Fig. 4. GPC profiles of carbohydrates in the acid phase after 2step phase-separation treatment

data. Analysis of carbohydrate composition and the ${ }^{1} \mathrm{H}-\mathrm{NMR}$ spectrum suggested that this fraction is water-soluble polymers containing glucose as major carbohydrate component, because the spectrum do not have aromatic ring signals (6-8 ppm) and presented many broad signals for carbohydrate (3-5 ppm). ${ }^{7}$

In the 2step phase-separation treatment, the carbohydrates in the sulfuric acid phase were higher molecular weight than the 1step phase-separation treatment. This difference of hydrolysis rate is based on the extraction effect of lignophenol from lignocellulosic with phenol derivatives.

3.3 Characterization of lignocresol

In order to investigate total material balance and characterization of lignocresol, lignocresol was purified from cresolic phase after the phase separation treatment. In the 1 step process the yield of lignocresol increased with the treatment time, the yield was $21.4 \%$ of raw material at $5 \mathrm{~min}$ treatment and $29.8 \%$ of raw material at $60 \mathrm{~min}$ treatment. In order to calculate the amount of original lignin, the amount of cresol combined with lignin was determined by ${ }^{1} \mathrm{H}-\mathrm{NMR}$. The amount of grafted cresol increased from 0.79 $\mathrm{mol} / \mathrm{C} 9$ to $0.85 \mathrm{~mol} / \mathrm{C} 9$ slightly by extending treatment time from $5 \mathrm{~min}$ to $60 \mathrm{~min}$. The molecular weight of lignocresol decreased to $\overline{\mathrm{Mw}}$ $=9400$ at $60 \mathrm{~min}$ from $\overline{\mathrm{MW}}=34000$ at $5 \mathrm{~min}$ treatment with the increase in grafted cresol. These results showed that depolymerization of 
lignin by cleavage of benzyl aryl ether proceed with the treatment and $p$-cresol is grafted at the same time.

On the other hand in the 2 step process, the yield of lignocresol was lower than the 1 step process. The molecular weights of lignocresol from 2 step process were higher than 1step process. These results show that the separation rate of lignocellulosics in the 1 step phase separation system is higher than the 2step phase separation system.

3.4 Phase-separation treatment with different hydrophilicity of phenol derivatives.

Conversion and separation patterns of lignocellulosics were investigated in the phase-separation treatments with different hydrophobic phenol derivatives $(2,4-x y l e n o l$, $p$-cresol, 4-methylcatechol).

The yields of carbohydrate from the sulfuric acid layer were $68 \%$ in 4-methylcatechol, $66 \%$ in $p$-cresol and $63 \%$ in 2,4-xylenol(Fig. 5). On the other hand, the yields of lignophenols from the phenol layer were $27 \%$ in 4 -methylcatechol and $p$-cresol, and $23 \%$ in 2,4-xylenol. The separation efficiency of lignocellulocics got higher with increasing hydrophilicity of the phenol became high because of the affinity of the phenol phase and acid phase.

It was possible to control conversion pattern of lignocellulosics component with the quantity of the phenol derivatives and the hydrophilicity of the phenol derivatives.

3.5 Total material balance of lignocellulosic components in the phase-separation treatment

In the phase-separation reaction system composed of phenol derivatives and concentrated acid, lignin and carbohydrates, which form an interpenetrating polymer network within the cell wall, were separated almost quantitatively into different phases within $20 \mathrm{~min}$ (Fig. 6). A total yield of lignocresol (cresol free) and sugars from the acid phase and the cresol phase was $96 \%$ of raw material at the $60 \mathrm{~min} 1 \mathrm{step}$ phase-separation treatment. In the 2 step process, the total yield was $88 \%$ of raw material at the $60 \mathrm{~min}$

In this system, because lignin was solvated with phenol derivative to prevent from the attack of concentrated acid, self-condensation of lignin was suppressed. Thus the belt effect of lignin to restrict carbohydrates hydrolysis was released, so that carbohydrates were rapidly swollen and dissolved in the concentrated acid.

Separated carbohydrates were consisted of various sizes of oligosaccharide and high molecular weight water-soluble glucose polymers. The water-soluble polysaccharide and oligosaccharide can be use for thickening agent etc. and functional food, respectively. These carbohydrate components can be converted readily to simple sugars by dilute acid hydrolysis. The monosaccharides also convert to ethanol or lactic acid through fermentation. The monosaccharides from hemicellulose are expected as pharmaceuticals, for example xylitol.

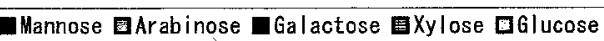

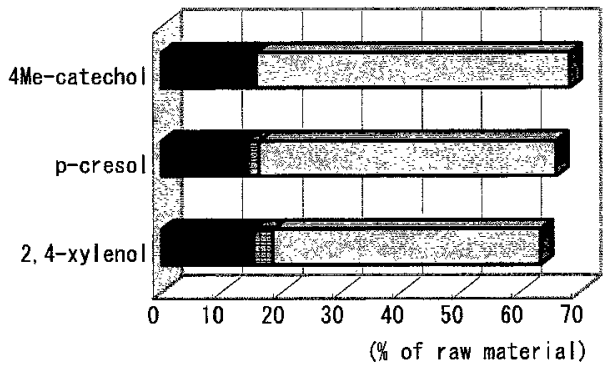

Fig. 5 Carbohydrate compositions of acid phase after the phase-separation treatment with different phenol derivatives

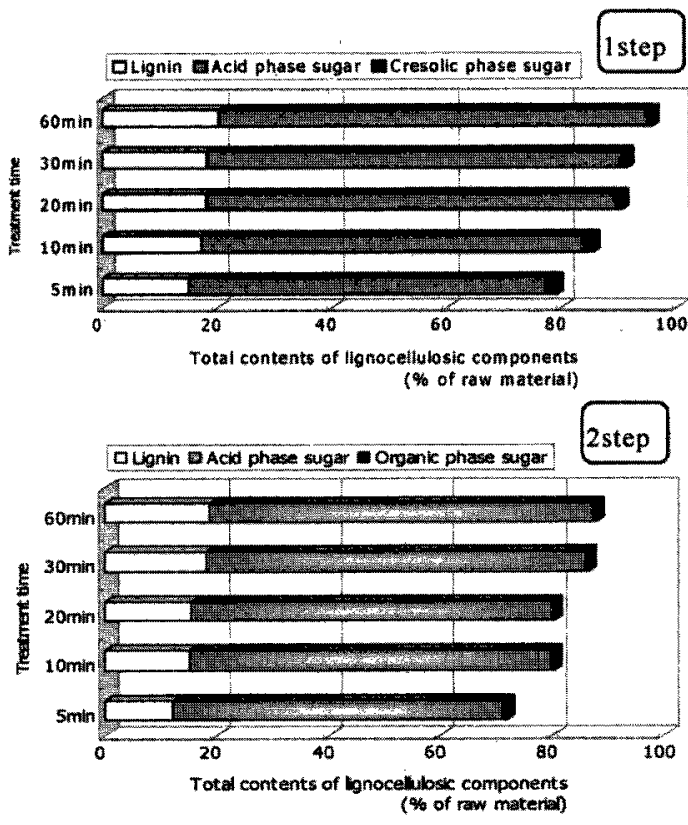

Fig. 6 Total material balance of lignocellulosic components in phase-separation treatment

4. References

[1] B. Johansson and G.E. Miksche, Acta. Chem. Scand. 23, 924 -930(1969)

[2] J.M. Harkin, Adv. Chem. Series 59, 65 (1966).

[3] S. Yasuda, N. Terashima and T. Ito, Mokuzai Gakkaishi 26, 552-557 (1980).

[4] M. Funaoka and I. Abe, Tappi Journal 72 145-149(1989).

[5] M. Funaoka and S. Fukatsu, Holzforschung 50, 245-252 (1996).

[6] M. Funaoka, Polymer International, 47, 277-290 (1998).

[7] L. A. Fluggue, J. T. Blank and P. A. Petillo, $J$. Am. Chem. Soc. 121, 7228-7238 (1999).

(Received February 16. 2007;Accepted July 12, 2007) 